See discussions, stats, and author profiles for this publication at: https://www.researchgate.net/publication/320920611

\title{
Image Enhancement Using Piecewise Linear Contrast Stretch Methods based on Unsharp Masking Algorithms for Leather Image Processing
}

Conference Paper · October 2017

DOI: $10.1109 /$ ICSITech.2017.8257197

CITATIONS

4

4 authors, including:

Murinto Murinto

Ahmad Dahlan University

57 PUBLICATIONS 80 CITATIONS

SEE PROFILE

Sri Winiari

Ahmad Dahlan University

15 PUBliCATIONS 19 CITATIONS

SEE PROFILE
1,697

A Adhi Prahara

Ahmad Dahlan University

14 PUBLICATIONS 13 CITATIONS

SEE PROFILE

Some of the authors of this publication are also working on these related projects:

Project Determining the nutrition of patient based on food packaging product using fuzzy $\mathrm{C}$ means algorithm View project

Applications of artificial intelligence and machine learning techniques and algorithms View project 


\title{
Image Enhancement Using Piecewise Linear Contrast Stretch Methods based on Unsharp Masking Algorithms for Leather Image Processing
}

\author{
${ }^{1}$ Murinto, ${ }^{2}$ Sri Winiarti, ${ }^{3}$ Dewi Pramudi Ismi, ${ }^{4}$ Adhi Prahara \\ Informatics Department \\ Universitas Ahmad Dahlan \\ Yogyakarta, Indonesia \\ $\underline{{ }^{1} \text { murintokusno@tif.uad.ac.id, }{ }^{2} \text { sri.winiarti@tif.uad.ac.id, }{ }^{3} \text { dewi.ismi@tif.uad.ac.id, }{ }^{4} \text { adhi.prahara@tif.uad.ac.id }}$
}

\begin{abstract}
This research work proposes a novel method to improve quality of animal leather images using digital image processing approach. In this work, piecewise linear contrast stretch based on unsharp masking algorithm is employed for image enhancement. The proposed method minimizes contrast problem. Experiments had been done on four categories of animal leather images namely crocodile leather, monitor lizard leather, cow leather and goat leather. The proposed method was then compared with other piecewise linear transforms based image enhancement techniques including intensity level slicing, bit plane slicing and contrast stretching methods. PSNR, MSE and SSIM values were obtained by using our proposed method and our proposed method produced better result. The values of PSNR when using piecewise linear contrast stretch unsharp masking (PLCSUS) respectively for crocodile leather, monitor lizard leather, cow leather and goat leather are $30.06 \mathrm{~dB}, 18.97$ $\mathrm{dB}, 20.66 \mathrm{~dB}$ and $14.73 \mathrm{~dB}$. This value is higher when compared to using other methods on the same image. Experiments show that our proposed method is better compared to conventional methods with respect to special characteristics of animal leather to be used as raw materials of artworks.
\end{abstract}

Keywords-contrast stretching; leather images; image enhancement; unsharp masking algorithm; piecewise linear transforms

\section{INTRODUCTION}

Small and medium enterprises in Indonesia experiences significant growth recently. One of them is leather craft industry. A leather craft industry has spread in many places in Indonesia, for example Sidoarjo, Cibaduyut, Yogyakarta, Magetan and other places outside Java Island. Bantul (a district in Yogyakarta Province) had a center of leather craft industry namely Manding leather craft zone. Leather craft in Manding produces various leather craft products such as gloves, belt, sandals, shoes, bags and other leather goods. Most of those products manufactured from cow leather, buffalo leather, goat leather, sheep leather and stingray skin, which are processed in home industry. Development of variations of types and designs of leather artworks in Manding is not balanced with the knowledge of their industry players. Leather industry practitioners in that area do not have knowledge about the standards of feasibility and scientific classification of processed leather. Knowledge of types and quality of leather to be used as leather craft raw materials is gained traditionally from generation to generation. The understanding of leather quality and authenticity and certain skin damage to craft products is very minimal. One common problem in craft production caused by this lack of knowledge is a certain craft product may be made of inappropriate raw materials. When such problem occurs, leather craft industry players incur loss because of unsound leather craft product produced.

There are many criteria to consider when selecting leather raw materials to create a certain leather craft. Therefore, determining appropriate raw materials for a certain leather craft requires sound knowledge on the materials. Initial step on this subject is to perform image enhancement on digital animal leather images prior to pattern recognition process on animal leather images. This is based on the fact that at the time of shooting animal leather images, the images cannot be used directly but they must go through image processing (image enhancement) first because the image obtained may have low contrast, too dark or too bright.

Image enhancement is one of the classic problems in image processing and pattern recognition. Image enhancement is widely used for image processing and is used as a preprocessing step in pattern recognition, classification, texture synthesis and many other image processing applications [1], [2], [3].Several image contrast enhancement algorithms are proposed in previous studies including gray level manipulation, filtering, histogram equalization (HE) and piecewise linear transformation, including: intensity level slicing, bit plane slicing, contrast stretching. Contrast is one of the important qualities in evaluating quality of images in subjective manner. Contrast is obtained from the difference in illumination reflected by two adjacent surfaces. In other words, contrast is a visual characteristic difference so that objects can be distinguished from their backgrounds. In visual perception, a contrast is determined through the difference in color and brightness of the object with other objects. Several algorithms for contrast enhancement have been developed and implemented in image processing problems. The main purpose of the image enhancement is to display the hidden detail in the image or to increase the contrast of a low contrast image [4]. 
The purpose of this study was to evaluate different image enhancement methods, especially piecewise linear transform methods, and compare them with our proposed method. Our proposed method is image enhancement method based on unsharp masking algorithms to be applied in leather image processing. The reason for using unsharp masking algorithms in this study is because unsharp masking algorithm [5] which is a halo effect analysis [6], and rescaling process requirements.

Moreover, the organization of this paper is as follows. In part I, the background of this study is presented. In part II it explains the image enhancement method which includes spatial domain and frequency domain along with image enhancement methods which are included. Part III is specifically explained about piecewise linear transforms methods that include intensity level slicing, bit plane slicing and contrast stretching methods. In section IV, it is explained about the unsharp masking algorithm framework and the proposed framework which includes the use of adaptive contrast stretching on contrast enhancement, low pass filter is replaced by bilateral filtering, and the use of adaptive gain control. In part $\mathrm{V}$ is explained about the use of Image Quality Measurement (IQM) which includes the subjective measurement and objective measurement. In Section VI presented the results of experiments conducted, comparing the results of image enhancement methods used in this study. Section VII presents conclusions and suggestions that can be done for further research.

\section{IMAGE ENHANCEMENT METHODS}

Image enhancement is a process to get an image becomes easier to be interpreted by human visually. Image enhancement can also be said to be a process for obtaining a better image for a particular application than the original image. The methods of image repair can be categorized into two parts, namely: methods that work on spatial domains and methods that work on the frequency domain.

In spatial domain - image enhancement method, the transformation is directly applied to the pixels. This method works on the whole pixel and can be written in (1).

$$
g(x, y)=T[f(x, y)]
$$

where $f(x, y)$ is the treated image, $g(x, y)$ is the processed image and $\mathrm{T}$ is the operator acting on $\mathrm{f}$. Spatial methods may also work on sub-images defined in a particular neighborhood area. In its implementation, is a window or mask is often used. The notion of a mask is a two-dimensional array with an element value selected according to the feature to be detected on an image. Some of the methods included in the spatial domain are: Gray Level Transformation [7], Image negative [8], Log transformation [9], Piecewise linear transformation including contrast stretching [10], intensity level slice [11], bit plane slice [12], Histogram Processing [13], Adaptive Power Transformation[14].

In frequency domain image enhancement method, the transformation is computed and then the enhancement method is applied. This method is based on convolution theory. Suppose $g(x, y)$ is the image obtained from the convolution image $f(x, y)$ with the position-invariant operator $h(x, y)$ i.e. $\mathrm{g}$ $(x, y)=h(x, y) * f(x, y)$ then from the theory of convolution obtained (2).

$$
G(u, v)=H(u, v) * F(u, v)
$$

Where $G, H, F$ is the Fourier transform of $g, h, f$. The function $H(u, v)$ is often referred to as a transfer function. The purpose of this process is to select $H(u, v)$ so that the desired image, $g(x, y)=F-1[H(u, v) F(u, v)]$, shows the feature $f(x$, $y)$. Example: Edge in $f(x, y)$ can be clarified by selecting the function $H(u, v)$ which accentuates the high frequency component at $f(x, y)$.

\section{PIECEWISE LINEAR TRANSFORMS METHODS}

In piecewise linear transform every pixel of the image will be manipulated. This transformation technique aims to improve quality of the image by changing the range of pixels intensity values in the original image. A common advantage of this technique is that changing complex functions can be solved using the piecewise linear method. There are three types of transformation: contrast stretching method, intensity level slicing method, bit plane slicing method [4].

\section{A. Contrast Stretching Method}

Contrast stretching is applied to the image to stretch the histogram to fill the full dynamic range of the image [4]. Two popular types of contrast stretching techniques are basic stretching contrast and end-in-search. Basic stretching contrast works well on the image where all the pixels concentrate in one part of the histogram, for example in the middle. Besides, contrast stretching is used to overcome deficiencies or excess light during shooting, extending the distribution of pixel-gray values, where images are usually grouped in: low contrast, fine contrast or normal contrast, and high contrast. The image with low contrast is characterized by most of its bright or mostly dark image composition. The histogram shows some degree of grayness in groups together. If the pixel grouping is on the left, then the image tends to be dark and vice versa. The image with low quality can be improved quality with contrast stretching operation. The contrast stretching algorithm is as follows:

1. Find the lower bound of pixel grouping by scanning the histogram of the smallest gray scale to the largest gray scale value ( 0 to 255 ) to create the first pixel that exceeds the predefined threshold value.

2. Find the upper border of pixel grouping by scanning the histogram of the largest gray scale value to the smallest value of the predefined second threshold value.

3. The pixels below the first threshold value are given a value of 0 , while the pixels above the second threshold value are 255.

4. The pixels between the first threshold value and the second scaled threshold value to satisfy the complete range of gray scale values ( 0 to 255 ) with the mathematical in (3).

$$
s=\frac{r-r_{\max }}{r_{\min }-r_{\max }} \times 255
$$


where $r$ is the gray scale value of the original image, $s$ is the new gray scale value, the lowest gray scale $r_{\min }$ value of the pixel group, the highest gray scale $r_{\max }$ of the pixel group.

\section{B. Intensity Level Slicing Method}

Intensity level slicing method often highlights a certain range of desired gray level imagery. The application includes improving certain features such as water masses in satellite imagery and flaws in X-ray imagery. There are two basic methods for level slicing. One is to display a high value on all gray levels there is a desired range and a low value for all other gray levels. Another approach is based on transformation, such as brightening the desired gray level range but still maintaining background and gray level tonalities in image [4].

\section{Bit Plane Slicing Method}

The low contrast image is strengthened using the image enhancement method. Often the image enhancement method brightens all pixels of input image. This weakness is usually overcome by using bit plane slicing. In bit-plane slicing, image is divided into eight parts of binary field [4]. Bits that are in bit plane 0 are categorized as least significant bits and the bits in plane 7 bit are categorized as the most significant bits. The intensity value of each pixel can be represented by an 8-bit binary vector $v_{i}\left(v_{7}, v_{6}, v_{5}, v_{4}, v_{3}, v_{2}, v_{1}, v_{0}\right)$, where $i$ is a value of 0 to 7 and each $v_{i}$ can have a value of ' 0 ' or ' 1 '. The equation of the bit plane can be written as (4).

$$
I_{\text {bit }}(i, j)=R\left\{\frac{1}{2} \text { Floor }\left[\frac{1}{2^{i}}, I(i, j)\right]\right\}
$$

where $I(i, j)$ is the original image, $I$ bit $(i, j)$ is the bit plane information, $R$ is remainder, Floor $(i)$ is the round of elements to the nearest integer less than equal to $i$.

\section{PROPOSED METHOD}

In this research we proposed method piecewise Linear Contrast Stretch Based on Unsharp Masking (PLCSUM) Method for leather image enhancement. Unsharp masking (UM) is an image manipulation technique. The unsharp masking technique can well improve detail appearance through small scale enhancements of edge contrast of an image. In general, unsharp mask is used to sharpen an image where this will help us to affirm image texture and image details. The classical unsharp masking algorithm can be written as (5).

$$
z=n+\gamma(m-n)
$$

Where $m$ is the input image, $n$ is result of the process using a linear low-pass filter, whereas $\gamma$ is the gain with $(\gamma>0)$ which is real scaling factor. Signal $d=m-n$ often amplifies $(\gamma>1)$ to increase sharpness. The signals consist of image details, noise, over-shoot and under-shoots in an area of sharp edge caused by smoothing edges.

When enhancement of a noise is not possible to perform, enhancements of over-shoot and under-shoots are obtained through a visually unpleasant halo effect. Here we need a filter that is not sensitive to noise and not smooth sharp edge.
Previous research works had used several known filters, including cubic filters and edge preserving filters to replace linear low-pass filters. However, both techniques possess some constraints. Cubic filter is not sensitive to noise, while edge preserving filter is not smooth sharp edge. Thus in this work, we proposed adaptive gain control. Fig. 1 shows block diagram of the classic unsharp masking algorithm which is used as the basis of unsharp masking algorithm proposed in this study. This paper will introduce an unsharp masking framework for leather image enhancement. This framework introduces a generalization of the unsharp masking algorithm combined with the operations of contrast stretching methods.

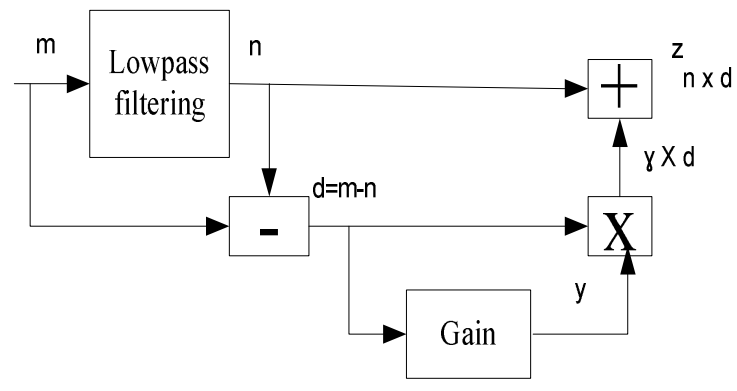

Fig.1. Block diagram of general unsharp masking algorithm.

This paper will introduce an unsharp masking framework for leather image enhancement. This framework is based on a generalization of the unsharp masking algorithm combined with the operations of adaptive contrast stretching on halo effect issues solved using an edge preserve filter. In this paper the edge preserve filter used bilinear filtering to generate the signal [15]. Bilinear filtering is selected due to its simplicity when compared to the median filter [16]. In this study the concept of enhancement and sharpening uses a different process that is using adaptive contrast stretching algorithm [17] and the output is called w (y). Image details are processed using $g(d)=\gamma(d) \otimes d$, where $d$ is an adaptive gain control and is a function of the amplitude of the detail signal of $d$. While the final result of the algorithm is written in (6).

$$
u=w(y) \oplus[\gamma(d) \otimes d]
$$

Frame piecewise linear contrast stretch based framework on unsharp masking algorithm is shown in Fig. 2.

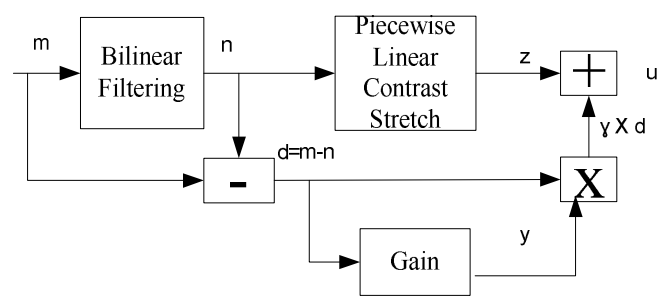

Fig.2. Framework piecewise linear contrast stretch based on unsharp masking algorithm. 


\section{IMAGE QUALITY MEASUREMENT}

There are two approaches to perform image quality measurement, namely: objective measurement and subjective measurement objective measurement and subjective measurement [18].

$$
S \operatorname{SIM}=\frac{(2 \mathrm{x} \overline{\mathrm{x}} \mathrm{x} \overline{\mathrm{y}}+\mathrm{C} 1)\left(2 \mathrm{x} \sigma_{\mathrm{xy}}+\mathrm{C} 2\right)}{\left(\sigma_{\mathrm{x}}^{2}+\sigma_{\mathrm{y}}^{2}+\mathrm{C} 2\right) \mathrm{x}\left((\overline{\mathrm{x}})^{2}+(\overline{\mathrm{y}})^{2}+\mathrm{C} 1\right)}
$$

where $\mathrm{C} 1$ and $\mathrm{C} 2$ are constants, $\bar{x}, \bar{y}, \sigma_{x}^{2}, \sigma_{y}^{2}$ and $\sigma_{x y}$ and $\sigma_{x y}$ are given as (10), (11), (12), (13), (14) respectively.

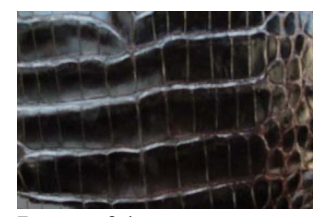

Image01

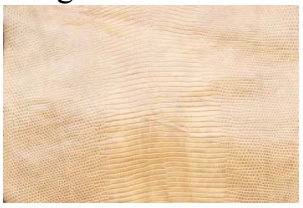

Image02

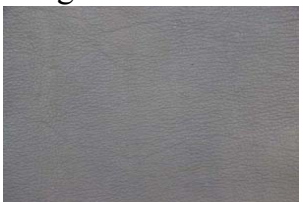

Image 03

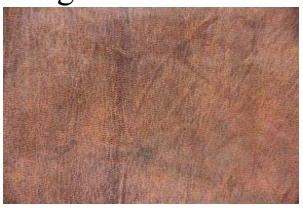

Image 04

(a)
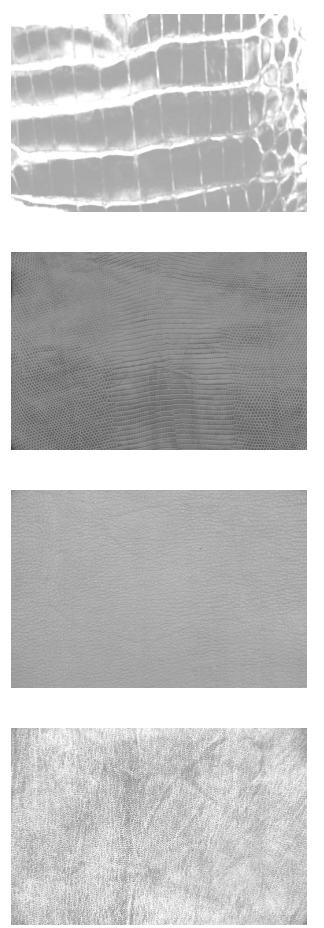

(b)
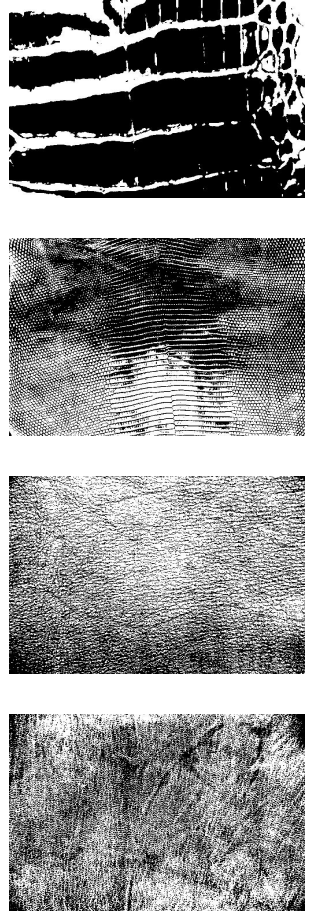

(c)
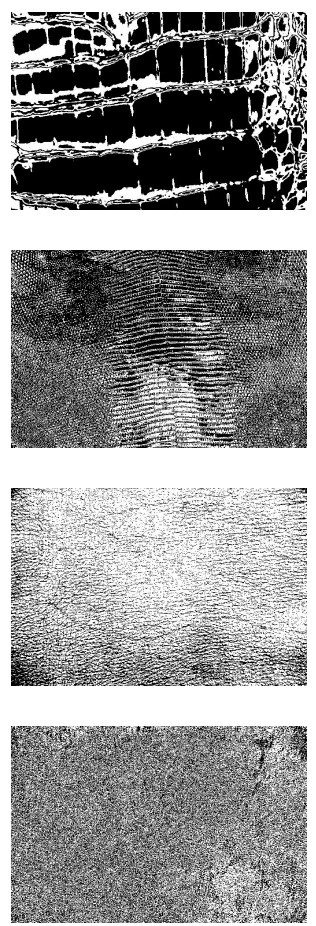

(d)
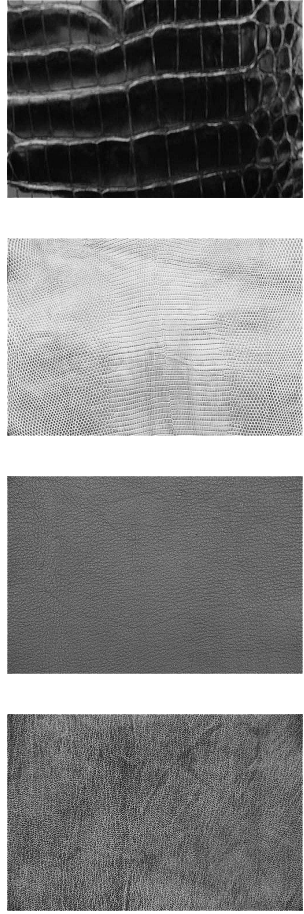

(e)

Fig.3.Image enhancement using different methods (a). Original images, (b) contrast stretching, (c) intensity level slicing, (d) bit plane slicing, (e) piecewise linear contrast stretch based on Unsharp Masking algorithm.

\section{A. Objective Quality Measurements}

Quality measures objectively use two measures: Peak Signal-to-Noise Ratio (PSNR). PSNR is an evaluation standard of reconstructed image quality. The small PSNR value of the image means that the image has a low quality. PSNR is evaluated in decibels. PSNR is defined as (7).

$$
\text { PSNR }=10 \log _{10} \frac{\left(2^{\mathrm{n}}-\right)^{2}}{\sqrt{\mathrm{MSE}}}
$$

Mean Squared Error (MSE)is defined as (8).

$$
\operatorname{MSE}=\frac{1}{M N} \sum_{i=1}^{M} \sum_{j=1}^{N}(x(i, j)-y(i, j))^{2}
$$

where $x(i, j)$ represents the original image (reference) and $y(i$, $j$ ) represents the distorted image and $i$ and $j$ represents the pixel position of the image $M x N$. The MSE will be zero when $x(i, j)=y(i, j)$.

\section{B. Subjective Quality Measurements}

One type of subjective quality measurement is to use SSIM (Structural Similarity Index Metric) defined as (9).

$$
\begin{gathered}
\bar{x}=\frac{1}{N} \sum_{i=1}^{N} x_{i} \\
\bar{y}=\frac{1}{N} \sum_{i=1}^{N} y_{i} \\
\sigma_{x}^{2}=\frac{1}{N-1} \sum_{i=1}^{N}\left(x_{i}-\bar{x}\right)^{2} \\
\sigma_{y}^{2}=\frac{1}{N-1} \sum_{i=1}^{N}\left(y_{i}-\bar{y}\right)^{2} \\
\sigma_{x y}=\frac{1}{N-1} \sum_{i=1}^{N}\left(x_{i}-\bar{x}\right)\left(y_{i}-\bar{y}\right)
\end{gathered}
$$

\section{EXPERIMENT RESULT}

\section{A. Image Dataset}

The image enhancement method introduced in this paper was tested on 4 different animal skin images. The four types of skin are crocodile skin of the body (image01), lizard skin (image02), cow skin (image03) and goat skin (image04). The image used in this research is 512 x 512 pixels. The original image is formatted RGB (*.bmp and *.jpg) and converted into grayscale image for easy processing. 


\section{B. Performance Comparison Image Enhancement}

In this study we compared intensity level slicing (ILS) methods, contrast stretching (CS), bit plane slicing methods (BPS) [4] and the proposed methods namely Piecewise Linear Contrast Stretch Based on Unsharp Masking (PLCSUM). The results obtained by using the four image enhancement methods are shown in Fig. 3. While the image quality measurement used PSNR, MSE and SSIM

In this research, image quality measurement is done through subjective and objective measurement. Objective evaluations used are mean squared error (MSE) and peaksignal-to-noise, as written in (7), (8). The subjective evaluations used are the SSIM (Structural Similarity Index Metric) as defined in (9). Four image enhancement methods applied to four types of animal skins are the crocodile skin of the back (image01), lizard skin (image02), cow skin (image03) and goat skin (image04). The application results of these methods are shown in Table I. PLCS is piecewise linear contrast stretching, ILSM is intensity linear slicing method, BPSM is bit plane slicing method and PLCSUS is piecewise linear contrast stretch unsharp masking.

TABEL I. PSNR, MSE, AND SSIM RESULTED FROM IMPLEMENTATION OF FOUR IMAGE ENHACEMENT METHODS

\begin{tabular}{|c|c|c|c|c|c|}
\hline Images & Index Quality & PLCS & ILSM & BPSM & $\begin{array}{c}\text { PLCSU } \\
\text { S }\end{array}$ \\
\hline image01 & PSNR (dB) & 4.05 & 9.19 & 3.67 & 30.06 \\
\hline & MSE & $\begin{array}{c}25785 . \\
75\end{array}$ & 7894.70 & 2736.64 & 64.57 \\
\hline & SSIM & 0.2669 & 0.0995 & 0.0214 & 0.9063 \\
\hline image02 & PSNR (dB) & 11.80 & 3.67 & 2.14 & 18.97 \\
\hline & MSE & 4328.0 & 28178.0 & 40008.23 & 830.21 \\
\hline & SSIM & 0.8335 & 0.1680 & 0.0004 & 0.6521 \\
\hline image03 & PSNR (dB) & 11.2 & 6.37 & 7.93 & 20.66 \\
\hline & MSE & 4720.5 & 15121.7 & 10562.85 & 563.09 \\
\hline & SSIM & 0.8510 & 0.1124 & 0.0081 & 0.5314 \\
\hline & PSNR (dB) & 7.92 & 7.37 & 7.84 & 14.73 \\
\hline & MSE & 10582 & 12004 & 10778.28 & 2203.36 \\
\hline & SSIM & 0.7770 & 0.2702 & 0.0016 & 0.5668 \\
\hline
\end{tabular}

In Table I, PNSR, MSE and SSIM values for different leather images is calculated. From Table III , we can see that the highest PSNR value is achieved when using the PLCSUS method for image processing i.e. $30.06 \mathrm{~dB}$ for image $01,18.97$ $\mathrm{dB}$ for image $02,20.66 \mathrm{~dB}$ for image $03,14.73 \mathrm{~dB}$ for image 04 . From this it can be concluded that method PLCSUS is better than the other method in leather image enhancement.

\section{CONCLUSIONS}

In this paper, a new technique for leather image enhancement has been proposed. The new technique uses piecewise linear contrast stretch based on unsharp masking algorithm. Experiments had been done on four animal leather images, namely crocodile leather image, lizard leather image, cow leather image and goat leather image. The PSNR, MSE and SSIM values obtained by using our proposed method show the best results compared to the other three image enhancement methods. The values of PSNR for piecewise linear contrast stretch unsharp masking respectively for crocodile leather, monitor lizard leather, cow leather and goat leather are 30.06 $\mathrm{dB}, 18.97 \mathrm{~dB}, 20.66 \mathrm{~dB}$ and $14.73 \mathrm{~dB}$. The higher the value of PSNR means that the image enhancement result produces better image quality compared to the original image. From this it can be concluded that method PLCSUS is better than the other method in leather image enhancement.

\section{ACKNOWLEDGMENT}

The researchers expressed gratitude to Institute for Research and Development (LPP) of Universitas Ahmad Dahlan Yogyakarta who has funded this research with Internal competitive research (PP) Scheme as outlined in the research contract number: PP-001 / SP3 / LPP-UAD / IV / 2017.

\section{REFERENCES}

[1] M. Righi, M. D’Acunto, and O. Salvetti, "An Image Enhancement Tool: Pattern Recognition Image Augmented Resolution", Journal Pattern Recognition and Image Analysis, Vol.26 No.3, 2016.

[2] Singh, Bikesh Kr. "Mammographic image enhancement, classification and retrieval using color, statistical and spectral Analysis." International Journal of Computer Applications 10 (2011): 18-23.

[3] Polesel, Andrea, Giovanni Ramponi, and V. John Mathews. "Image enhancement via adaptive unsharp masking." IEEE transactions on image processing 9.3 (2000): 505-510.

[4] Rafael C. Gonzalez, Richard E. Woods, "Digital Image Processing”, 2nd edition, Prentice Hall, 2002.

[5] G. Ramponi, "A cubic unsharp masking technique for contrast enhancement," Signal Process., pp. 211-222, 1998.

[6] Z. Farbman, R. Fattal, D. Lischinski, and R. Szeliski, "Edge-preserving decompositions for multi-scale tone and detail manipulation," $A C M$ Trans. Graph., vol. 27, no. 3, pp. 67:1-67:10, 2008.

[7] Raji, A., et al. "A gray-level transformation-based method for image enhancement." Pattern Recognition Letters 19.13 (1998): 1207-1212.

[8] Maini, Raman, and Himanshu Aggarwal. "A comprehensive review of image enhancement techniques." arXiv preprint arXiv:1003.4053 (2010).

[9] Bedi, S. S., and Rati Khandelwal. "Various image enhancement techniques-a critical review." International Journal of Advanced Research in Computer and Communication Engineering 2.3 (2013).

[10] Arici, Tarik, Salih Dikbas, and Yucel Altunbasak. "A histogram modification framework and its application for image contrast enhancement." IEEE Transactions on image processing 18.9 (2009): 1921-1935.

[11] Fraz, Muhammad Moazam, et al. "An approach to localize the retinal blood vessels using bit planes and centerline detection." Computer methods and programs in biomedicine 108.2 (2012): 600-616.

[12] Vij, Komal, and Yaduvir Singh. "Enhancement of images using histogram processing techniques." Int. J. Comp. Tech. Appl 2.2 (2009): 309-313.

[13] Stark, J. Alex. "Adaptive image contrast enhancement using generalizations of histogram equalization." IEEE Transactions on image processing 9.5 (2000): 889-896.

[14] Srinivasan, S., and N. Balram. "Adaptive contrast enhancement using local region stretching." Proc. of ASID. Vol. 6. 2006.

[15] C. Tomasi and R. Manduchi, "Bilateral filtering for gray and color images," in Proc. IEEE ICCV, Jan. 1998, pp. 839-846.

[16] M. Fischer, J. L. Paredes, and G. R. Arce, "Weighted median image sharpeners for the world wide web," IEEE Trans. Image Process., vol. 11, no. 7, pp. 717-727, Jul. 2002.

[17] Dijk, Judith, et al. "Local adaptive contrast enhancement for color images." Visual Information Processing. 2007.

[18] Ahmet M. Eskicioglu, Paul S. Fisher, "Image Quality Measures and Their Performance" IEEE Transactions on Communication, Vol. 43, No. 12, pp. 2959-2965, December 1995. 